\title{
Producción de Bioetanol a partir de Elodea sp.
}

\author{
Tatiana Marulanda López \\ Programa de Ingeniería Ambiental. \\ Universidad de San Buenaventura- \\ Medellín \\ tmarulandalopez@gmail.com
}

\author{
Luisa Fernanda Zapata Osorno \\ Programa de Ingeniería Ambiental. \\ Universidad de San Buenaventura- \\ Medellín \\ luisafda-16@hotmail.com
}

\author{
María Consuelo Jaramillo Flórez \\ Programa de Ingeniería Ambiental. \\ Universidad de San Buenaventura- \\ Medellín \\ mariac.jaramillo@usbmed.edu.co
}

(Tipo de Artículo: Investigación Científica y Tecnologica. Recibido el 30/09/2016. Aprobado el 13/02/2017)

\begin{abstract}
Resumen. La dependencia de los países a los combustibles fósiles es cada vez mayor, pero estos no son renovables. Además, el uso de estos combustibles ha aumentado la emisión de gases de efecto invernadero. Colombia debe empezar a buscar otras alternativas que suplan la deficiencia en combustible, y cuya fuente de obtención sea renovable y no comprometa los recursos agrícolas usados como alimentos, tal es el caso de la caña de azúcar. Las plantas invasoras se caracterizan por producir eutrofización de las aguas, ocasionando problemas ambientales y de abastecimiento de agua a las ciudades. El manejo que se hace de esta planta es erradicarla usando diferentes métodos. En este artículo se muestra el aprovechamiento de la planta invasora de cuerpos acuosos, Elodea sp., para la obtención de bioetanol. Después de la hidrólisis de los carbohidratos, la detoxificación, y la fermentación, la planta mostró un alto rendimiento en la producción de bioetanol. Este trabajo es la base para el desarrollo del bioproceso, usando como sustrato el material lignocelulítico, y además, es una alternativa para el manejo de la planta Elodea sp. bomo fuente de energía carburante.
\end{abstract}

Palabras clave. Bioetanol, Detoxificación, Elodea sp., fermentación, Levadura, Saccharomycess cerevisiae.

\section{Production of Bioethanol from Elodea sp.}

\begin{abstract}
The dependence of countries on fossil fuels is increasing, but these are not renewable. In addition, the use of these fuels has increased the emission of greenhouse gases. Colombia must begin to look for other alternatives that overcome the deficiency in fuel, and whose source of production is renewable and this alternative does not compromise the agricultural resources used as food, such is the case of sugar cane. Invasive plants are characterized by eutrophication of water, causing environmental and water supply problems to the cities. The management of this plant is to eradicate it using different methods. This article shows the use of the invading plant of aqueous bodies, Elodea sp., for the production of bioethanol. After hydrolysis of carbohydrates, detoxification, and fermentation, the plant showed a high yield in the production of bioethanol. This work is the basis for the development of the bioprocess, using lignocellulose material as a substrate and, in addition, it is an alternative for the management of the Elodea sp. as a source of fuel energy.
\end{abstract}

Keywords. Bioethanol, detoxification, Elodea sp., fermentation, yeast, Saccharomyces cerevisiae. 


\section{Introducción}

Existen diferentes fuentes de energía: solar, eólica, geotérmica, de hidrógeno y biomasa, que son usadas para suplir la demanda energética a nivel mundial. Los combustibles fósiles como el petróleo, el diésel o el aceite crudo son recursos no renovables. Uno de los mayores problemas económicos a nivel mundial y regional es la importación de petróleo y sus derivados, debido a que muchos países son dependientes de estos recursos y por ende de los países que los producen. El aumento del costo y la disminución de los combustibles fósiles, además de la inestabilidad política en muchos países, obliga a que se busquen alternativas de obtención de energía [1], [2], [3]. El mayor reto a nivel mundial, además de buscar alternativas para sustituir los combustibles fósiles, es reducir las emisiones de los gases emitidos por estos, conservar el medio ambiente, y contribuir al desarrollo socioeconómico en áreas rurales [1]. Los combustibles fósiles emiten gases como $\mathrm{CO}, \mathrm{CO}_{2}, \mathrm{NOx}$, e Hidrocarburos Aromáticos Policíclicos, $\mathrm{PAH}$ [1], [2], [3]. Las altas concentraciones de NOx causan neumonía, irritación pulmonar, edemas, asma y bronquitis. Las emisiones de $\mathrm{CO}$ desarrollan tumores en niños y fetos. Las emisiones de PAH aumentan la morbilidad de personas con problemas respiratorios. Los $\mathrm{PAH}$ producen somnolencia, irritación ocular, flujo nasal $y$ tos. Las enfermedades pulmonares son causadas por hidrocarburos originados de la combustión de diésel. El ozono troposférico, originado por la combustión de NOx y compuestos orgánicos volátiles de la combustión del diésel, afecta la salud y causa daños al ecosistema, representado en necrosis del tejido vegetal, además de causar daño a los seres humanos. La acidificación de las lluvias es causada por las emisiones de los NOx y de sulfuro cuando reaccionan con el vapor de agua de la atmósfera, formando ácido nítrico, ácido nitroso, ácido sulfúrico, ácido sulfhídrico, estos ácido perjudican la vegetación, los materiales con los que se construyen los edificios produciendo un envejecimiento de estos, y los ecosistemas acuáticos y terrestres en general [2], [3], [4], [5], [6].

La bioenergía se presenta como una opción para mitigar el cambio climático, y, además, como fuente renovable que puede reemplazar los combustibles fósiles en el mercado energético, usando la bioenergía en la producción de calor, electricidad, y combustible para el transporte. La fuente para la producción de esta bioenergía o biocombustible se basa, en la actualidad, en la utilización de fuentes de alimentos como caña de azúcar, maíz, trigo, papa, etc, cuyo proceso está bien definido y completamente comercializado [7]. En lo que respecta a Colombia, el alcohol carburante que se produce, proviene exclusivamente del procesamiento de caña de azúcar del Valle Geográfico del Río Cauca, una zona rica cañera a nivel mundial [8]. Una amplia variedad de tecnologías de conversión están siendo construidas, que son de bajo costo y amigables con el medio ambiente [9], [10].
Existen algunas plantas denominadas plantas invasoras acuáticas. Estas se caracterizan por crecer en medios acuosos, y rápidamente se distribuyen por todo el sistema, provocando pérdida de la biodiversidad y ruptura de la funcionalidad del ecosistema, ya que disminuye la riqueza, densidad y biodiversidad de las plantas nativas, aumenta la mortalidad de animales acuáticos por eutrofización de las aguas, y ocasiona problemas en el abastecimiento del agua a las ciudades, en caso de encontrarse en represas [11]. La Elodea sp. es una planta acuática considerada invasora en muchas partes del mundo, debido al rápido crecimiento que tiene en aguas frías de climas tropicales, pues sus raíces están en la capacidad de crecer en cualquier parte del tallo, permitiéndole sujetarse con facilidad del suelo [12], [13], [14], [15]. Debido a los problemas ecológicos y económicos que esta planta crea, se han implementado varios métodos de control, tales como reducción de nutrientes en el medio, métodos mecánicos como corte, dragado, y métodos biológicos como introducción de peces herbívoros, o aplicando herbicidas [12]. Esta planta ha sido usada para remediar metales pesados, y una alternativa de utilización de esta planta es como fuente de obtención de bioetanol [16], [17], [18], [19].

La planta Elodea sp.es considerada una plaga al obstruir los sistemas de drenaje [19], por lo que este trabajo muestra una alternativa de utilización de esta planta, y el rendimiento del proceso de fermentación de la planta Elodea sp. en la obtención de bioetanol.

\section{Metodología}

La metodología consistió en secar la planta, obtener los azúcares fermentables detoxificados, someter a fermentación y analizar el contenido de etanol obtenido por espectrofotometría [20], [21], [22], [23]. El etanol glucosa, sacarosa, fenol, $\mathrm{K}_{2} \mathrm{Cr}_{2} \mathrm{O}_{7}, \mathrm{NaOH}$ anhidro, $\mathrm{H}_{2} \mathrm{SO}_{4}$ y los demás reactivos fueron adquiridos grado reactivo.

\subsection{Microorganismo}

La levadura Sacchraromyces cerevisiae deshidratada fue obtenida comercialmente en la ciudad.

\subsection{Medio de Fermentación}

El medio de fermentación contenía: glucosa $75 \mathrm{~g} / \mathrm{L}$; $\mathrm{NH}_{4} \mathrm{Cl} 10 \mathrm{~g} / \mathrm{L} ; \quad \mathrm{NaH}_{2} \mathrm{PO}_{4} \quad 0,5 \mathrm{~g} / \mathrm{L} ; \mathrm{NaCl} \quad 0,4 \mathrm{~g} / \mathrm{L} ;$ $\mathrm{MgCl}_{2}{ }^{*} 6 \mathrm{H}_{2} \mathrm{O} 0,35 \mathrm{~g} / \mathrm{L} ; \mathrm{CaCl}_{2}{ }^{*} 2 \mathrm{H}_{2} \mathrm{O} 0,05 \mathrm{~g} / \mathrm{L}$; levadura 15 $\mathrm{g} / \mathrm{L}$. Se calentó a $303 \mathrm{~K}$ y el pH fue llevado entre 5 y 6.

\subsection{Recolección, acondicionamiento e hidrólisis de la lignocelulosa}

La planta Elodea sp. fue adquirida en una represa del oriente Antioqueño, fue secada a $323 \mathrm{~K}$ por $24 \mathrm{~h}$ y triturada.

Se tomaron $60.0 \mathrm{~g}$ de material vegetal y se sometieron al calentamiento en soxhlet por $2 \mathrm{~h}$ en una solución de $\mathrm{H}_{2} \mathrm{SO}_{4} 0.1 \mathrm{~mol} / \mathrm{L}$. La mezcla obtenida se detoxifica para eliminar compuestos fenólicos. 


\subsection{Detoxificación de los azúcares fermentables}

La solución hidrolizada anteriormente obtenida se calentó a $333 \mathrm{~K}$, se le adicionó $\mathrm{NaOH}$ hasta $\mathrm{pH} 8.0$ y se agregó $\mathrm{Ca}(\mathrm{OH})_{2}$ sólido hasta $\mathrm{pH} 10$, para precipitar compuestos inhibidores de la levadura. La solución detoxificada se filtró y se llevó a pH entre 5 y 6 .

\subsection{Etapa de fermentación}

Se preparó la fermentación de la planta Elodea sp. con la solución de azúcares fermentables detoxificada y el medio de fermentación hasta un volumen total de 300 $\mathrm{mL}$ de tal forma que la concentración de levadura fuera $0.1 \mathrm{~g} / \mathrm{mL}$. Se dejó incubar por 2 días a temperatura ambiente. Transcurrido el tiempo se filtró el fermento, para someterlo a destilación. La fermentación se realizó por triplicado. La fermentación control se preparó adicionando agua en vez del extracto de la planta. El control se realizó por triplicado.

\subsection{Determinación de carbohidratos}

Se determinó por espectrofotometría (espectrofotómetro Genesys 10S UV-Vis, Thermo Scientific), midiendo el color generado por el hidroximetilfurfural, HMF, a $430 \mathrm{~nm}$, que se forma al mezclar la solución de fenol al $0.05 \mathrm{~g} / \mathrm{mL}$ y $\mathrm{H}_{2} \mathrm{SO}_{4}$ concentrado a la solución de azúcares. La curva de calibración se realiza preparando una solución stock compuesta por $1 \mathrm{~g} / \mathrm{mL}$ de glucosa y $1 \mathrm{~g} / \mathrm{mL}$ de sacarosa, y realizando diluciones sucesivas. Cada dilución de carbohidrato y la reacción se prepararon por triplicado.

\subsection{Determinación de la concentración de bioetanol}

Se determinó usando el método espectrofotométrico indirecto, por determinación de $\mathrm{Cr}^{3+}$ a $600 \mathrm{~nm}$, por reacción de oxidación de $10 \mathrm{ml}$ de bioetanol destilado con $5 \mathrm{~mL}$ de $\mathrm{K}_{2} \mathrm{Cr}_{2} \mathrm{O}_{7}$ a $0.5 \mathrm{~mol} / \mathrm{L}$ y $2 \mathrm{~mL} \mathrm{H}_{2} \mathrm{SO}_{4}$ concentrado a $333 \mathrm{~K}$ por media hora. La curva de calibración del etanol se realizó a partir de una solución stock de $0.1 \mathrm{~mL}$ etanol $/ \mathrm{mL}$ solución de etanol, con diluciones sucesivas. Cada dilución se preparó por triplicado. Las lecturas se realizaron por triplicado a 301 $\mathrm{K}$.

\subsection{Análisis estadístico}

Los resultados de la curva de calibración de carbohidratos y etanol, y de las muestras de la concentración de etanol y de carbohidratos en la fermentación y en la muestra detoxificada se presentaron como la Media \pm SEM del triplicado de las muestras. Los datos fueron analizados usando el test de análisis de varianza de Student Newman Keuls con GraphPad Prism 5,0 (GraphPad software, 2.007, San Diego, CA, USA). El valor de P menor de 0.05 fue considerado como significativo.

\section{Resultados y discusión}

Ingenierías USBMed, Vol. 8, No. 1, Enero-Junio 2017

\subsection{Determinación de las curvas de calibración de carbohidratos y etanol}

La curva de calibración se realizó graficando la absorbancia vs la concentración de carbohidratos. La Figura 1, muestra la gráfica de la curva de calibración de carbohidratos, de donde se obtiene la ecuación.

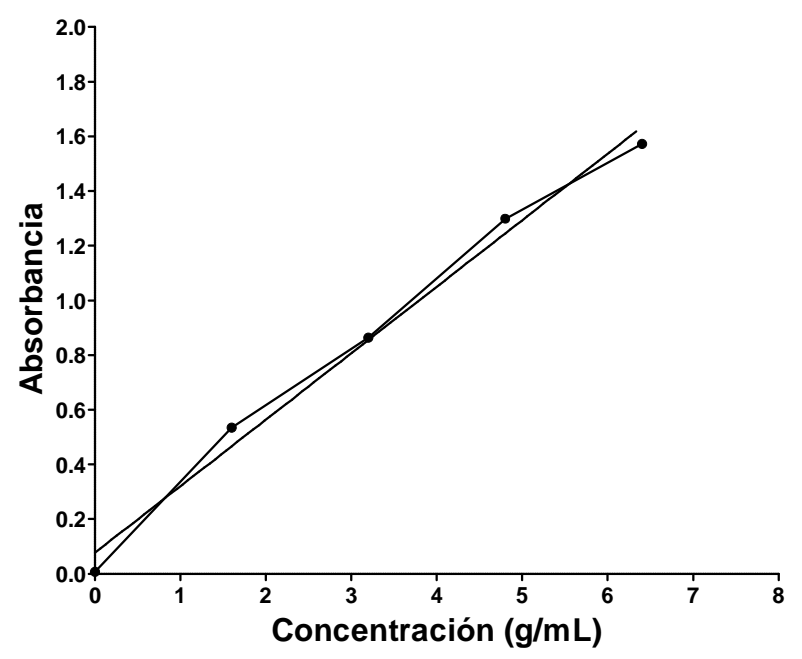

Figura 1. Curva de calibración de carbohidratos

La ecuación (1) muestra la curva de calibración de carbohidratos, para la obtención de la concentración de carbohidratos después de la detoxificación de los azúcares fermentables obtenidos de la planta Elodea sp. La curva de calibración arroja un coeficiente de correlación de $\mathrm{R}^{2}=0.9893$, con un valor de $\mathrm{P}$ menor de 0.0005 , lo que es considerado como significativo.

La curva de calibración de etanol se realizó graficando la Absorbancia vs la concentración de etanol. La Figura 2 , muestra la gráfica de la curva de calibración de etanol.

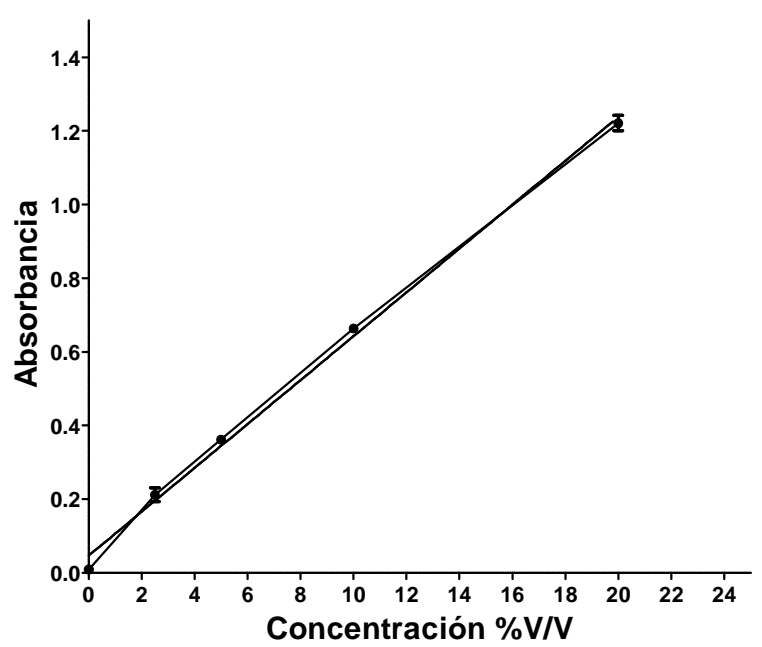

Figura 2. Curva de calibración de etanol

La ecuación (2) muestra la curva de calibración de etanol para la obtención de la concentración de bioetanol obtenido de la fermentación de la planta. Esta ecuación 
arrojó un coeficiente de correlación de $\mathrm{R}^{2}=0.9969$, con un valor de $\mathrm{P}$ menor de 0.0001 , lo que es considerado como significativo.

$$
\begin{aligned}
& Y=(0.2431 \pm 0.01457) X+(0.07735 \pm 0.05712) \\
& Y=(0.05955 \pm 0.001906) X+(0.04698 \pm 0.01964)
\end{aligned}
$$

\subsection{Determinación de la concentración de carbohidratos y etanol}

Las concentraciones de etanol obtenido en el proceso, y carbohidratos residuales se hallaron con las ecuaciones de las curvas de calibración. Antes de realizar la fermentación se determinó la concentración de carbohidratos en el extracto fermentable detoxificado. De acuerdo a las anteriores ecuaciones se obtuvo que la concentración de azúcares fermentables de la planta después de la detoxificación de la solución de Elodea sp. fue $1.96 \mathrm{~g} / \mathrm{mL}$, como se muestra en la Tabla 1 , y la concentración de etanol obtenido fue $0.18 \%(\mathrm{~V} / \mathrm{V})$, obtenido por la resta entre la concentración de etanol de la planta y el control.

\section{Tabla 1. Concentración de carbohidratos y etanol}

\begin{tabular}{lll}
\hline Muestra & & Concentración \\
\hline $\begin{array}{l}\text { Concentración de } \\
\text { Control }(\mathrm{g} / \mathrm{mL})\end{array}$ & \multicolumn{1}{c}{ Carbohidratos del } & $1.2237 \pm 0.1236$ \\
\hline $\begin{array}{l}\text { Concentración de Carbohidratos } \\
\text { después de la detoxificación de la } \\
\text { Elodea sp. }(\mathrm{g} / \mathrm{mL})\end{array}$ & \\
\hline $\begin{array}{l}\text { Concentración de Etanol producido por } \\
\text { la fermentación del control }(\% \mathrm{~V} / \mathrm{V})\end{array}$ & $4.3981 \pm 0.0412$ \\
\hline $\begin{array}{l}\text { Concentración de Etanol producido por } \\
\text { la fermentación de la planta }(\% \mathrm{~V} / \mathrm{V})\end{array}$ & $4.5782 \pm 0.2300$ \\
\hline $\begin{array}{l}\text { Concentración de etanol producido por } \\
\text { la planta Elodea sp. }(\% \mathrm{~V} / \mathrm{V})\end{array}$ & 0.18 \\
\hline Rendimiento de la fermentación & $14.0 \%$ \\
\hline
\end{tabular}

El rendimiento en el proceso de fermentación de la planta Elodea sp. fue del $14.0 \%$ después del proceso de fermentación. Este fue determinado con la relación entre la concentración de etanol producido por la planta Elodea sp. y el etanol teórico que se hubiese producido por la concentración de carbohidratos en la planta después de la detoxificación, que para este caso sería $1.2738 \mathrm{~mL}$ etanol $/ \mathrm{mL}$ solución.

El rendimiento en la fermentación es el esperado teniendo en cuenta la concentración de carbohidratos iniciales de la planta y el contenido de carbohidratos que tiene las plantas acuáticas. La concentración de carbohidratos solubles y almidón reportados en la planta Elodea nuttalli varía de acuerdo a la época del año, se reporta entre $4 \mathrm{mg} / \mathrm{g}$ de peso seco y $19 \mathrm{mg} / \mathrm{g}$ de peso seco de carbohidratos, y entre $3 \mathrm{mg} / \mathrm{g}$ y $37 \mathrm{mg} / \mathrm{g}$ de peso seco de almidón [24], además la concentración de carbohidratos solubles varía de acuerdo a la concentración de urea del medio en el que se encuentra la planta, alcanzando una concentración máxima de 20 $\mathrm{mg} / \mathrm{g}$ de peso seco al estar en un medio que contiene 5 $\mathrm{mg} / \mathrm{L}$ de urea [25]. El contenido de proteína es mayor en estas plantas, $367 \mathrm{mg} / \mathrm{g}$ de peso seco, y depende igualmente del mes del año en el cual se determine [24], por lo tanto es usada como fuente de nutrición [26].

La cantidad de agua obtenida en el proceso de destilación es un factor importante a tener en cuenta para la determinación de la concentración de etanol carburante, el etanol debe ser sometido a secado para la lectura de la concentración en el espectrofotómetro, y por ende para ser utilizado como biocombustible.

El contenido de lignina que contiene la planta debería ser hidrolizada para aumentar el rendimiento en el proceso de fermentación. La hidrólisis ácida usada en este método poco solubiliza la lignina por lo cual se somete a hidrólisis básica. La lignina está compuesta por derivados del alcohol cumarílico, compuestos tóxicos para la levadura, por lo que se deben precipitar [22]. La hidrólisis ácida solubiliza y despolimeriza la celulosa y le hemicelulosa convirtiéndose en la fuente de sustrato para la levadura [22].

Después de los dos días de fermentación se determinó la concentración de carbohidratos en el control y en la planta. La Tabla 2 muestra la concentración de carbohidratos sin fermentar del control y de la planta después de la fermentación.

Tabla 2. Concentración de carbohidratos después de la fermentación.

\begin{tabular}{ll}
\hline Muestra & Concentración $(\mathrm{g} / \mathrm{mL})$ \\
\hline $\begin{array}{l}\text { Concentración de Carbohidratos } \\
\text { del Control }\end{array}$ & $0.0023 \pm 0.0434$ \\
\hline $\begin{array}{l}\text { Porcentaje de carbohidratos no } \\
\text { fermentados en el control }\end{array}$ & $0.19 \%$ \\
\hline $\begin{array}{l}\text { Concentración de carbohidratos } \\
\text { en la planta Elodea sp. }\end{array}$ & $0.4374 \pm 0.0282$ \\
\hline $\begin{array}{l}\text { Porcentaje de carbohidratos no } \\
\text { fermentados en la planta Elodea }\end{array}$ & $22 \%$ \\
sp.
\end{tabular}

El porcentaje de carbohidratos en el control y en los azúcares fermentables, después de la fermentación, se calculó comparando los resultados con la concentración de carbohidratos iniciales, antes de la fermentación. Para el caso del control solo quedó el $0.19 \%$ de carbohidratos sin fermentar después de dos días de fermentación, y el porcentaje de azúcares no fermentados en la planta Elodea sp. fue del $22 \%$. Estos resultados muestran que el tiempo de fermentación no fue suficiente o la capacidad fermentadora de la levadura no es suficiente. Se requiere una mayor activación de la herramienta bioquímica de la levadura antes de ser sometida a las condiciones del experimento.

Es de destacar que el producto de la fermentación es tóxico para la levadura [15], lo que se debe tener en cuenta para el rediseño de la fermentación, y explicaría el exceso de carbohidratos sin fermentar al final del proceso. El mismo producto del metabolismo de la levadura es el responsable de la inhibición de esta, y por consiguiente de la fermentación. Además, hasta ahora no se reporta el uso de esta planta como fuente de bioetanol. El contenido químico de las plantas depende 
del sitio y de la época de recolección, habría que recolectar esta planta de otras zonas acuíferas para determinar el rendimiento en el proceso de fermentación en la obtención de la concentración de bioetanol [24].

\section{Conclusiones}

La crisis que la industria del petróleo enfrenta en la actualidad obliga a que todos los países busquen nuevas alternativas para la obtención de energía.

El porcentaje de etanol obtenido en el proceso de fermentación de la planta Elodea sp. muestra a esta planta como potencial fuente de obtención de biocombustible.

Se debe tener en cuenta la cantidad de agua obtenida en el proceso de destilación, ya que el poder carburante del etanol aumenta en ausencia de agua, lo que obliga a la búsqueda de métodos de destilación y procesos de secado más óptimos, donde se obtenga menor cantidad de agua.

Nuestro grupo de investigación continúa en la búsqueda de alternativas renovables de obtención de biocombustibles y en la optimización del proceso de fermentación, y así contribuir al desarrollo de nuestro país.

\section{Trabajos futuros}

Este trabajo presenta como alternativa de obtención de biocombustible, el uso de la planta Elodea sp. Para obtener un mayor rendimiento en la obtención de biocombustible se diseñará un biorreactor para optimizar la fermentación. La levadura se inmovilizará en un soporte apropiado, con el fin de que el mismo etanol obtenido en la fermentación y los compuestos tóxicos originados en el proceso, no afecte la viabilidad de la levadura.

\section{Agradecimientos}

Los autores agradecen el apoyo de la Universidad de San Buenaventura Medellín, por el apoyo en este trabajo.

\section{Referencias}

[1] Azad, A.K. M.G. Rasul, M.M.K. Khan, Subhash C. Sharma, M.A. Hazrat. «Prospect of biofuels as an alternative transport fuel in Australia,» Renewable and Sustainable Energy Reviews, vol. 43, pp. 331-351, 2015.

[2] Mofijur, M. M.G. Rasul, J. Hyde, M.M.K. Bhuyia. «Role of Biofuels on IC Engines Emission Reduction,» Energy Procedia, vol. 75, pp. 886892, 2015.

[3] Mofijur, M., M.G. Rasul, J. Hyde, A.K. Azad, R. Mamat, M.M.K. Bhuiya. «Role of biofuel and their binary (diesel-biodiesel) and ternary (ethanol-biodiesel-diesel) blends on internal combustion engines emission reduction,» Renewable and Sustainable Energy Reviews, vol. 53, pp. 265-278, 2016.

[4] Laj, P., J. Klausen, M. Bilde, C. Plaß-Duelmer, G. Pappalardo, C. Clerbaux, U. Baltensperger, J. Hjorth, D. Simpson, S. Reimann, P.-F. Coheur, A. Richter, M. De Maziere, Y. Rudich, G. McFiggans, K. Torseth, A. Wiedensohler, S. Morin, M. Schulz, et al. «Measuring atmospheric composition change,» Atmospheric Environment, vol. 43, p. 5351-5414, 2009.

[5] Holopainen, Riikka Rinnan and Toini. «Ozone Effects on the Ultrastructure of Peatland Plants: Sphagnum Mosses, Vaccinium oxycoccus, Andromeda polifolia and Eriophorum vaginatum,» Annals of Botany, vol. 94, p. 623-634, 2004.
[6] Schneider, Gerald F., Alexander W. Cheesman, Klaus Winter, Benjamin L. Turner, Stephen Sitch, Thomas A. Kursar. «Current ambient concentrations of ozone in Panama modulate the leaf chemistry of the tropical tree Ficus insipida,» Chemosphere, vol. 172, pp. 363-372, 2017.

[7] Gupta, Vijai K., Ravichandra Potumarthi, Anthonia O’Donovan, Christian P. Kubicek, Gauri Dutt Sharma, Maria G. Tuohy. «Chapter 2 - Bioenergy Research: An Overview on Technological Developments and Bioresources,» Bioenergy Research: Advances and Applications, pp. 23-47, 2014.

[8] Semana, Revista, «La inestable apuesta por los biocombustibles,» Revista Semana, 2002 2016. [En línea]. Available: http://www.semana.com/economia/articulo/biocombustibles-ya-noson-tan-buen-negocio/461232. [Último acceso: 0702 2017].

[9] Naveen Kumar Mekala, Ravichandra Potumarthi, Rama Raju Baadhe, Vijai K. Gupta. «Chapter 1 - Current Bioenergy Researches: Strengths and Future Challenges,» Bioenergy Research: Advances and Applications, pp. 1-21, 2014.

[10] Luiz J. Visioli, Fabiane M. Stringhini, Paulo R.S. Salbego, Daniel P. Chielle, Gabrielly V. Ribeiro, Juliana M. Gasparotto, Bruno C. Aita, Rodrigo Klaic, Jéssica M. Moscon, Marcio A. Mazutti. «Chapter 3 - Use of Agroindustrial Residues for Bioethanol Production,» Bioenergy Research: Advances and Applications, pp. 49-56, 2014.

[11] Shackleton, Ross T., David C. LeMaitre, BrianW. VanWilgen, David M. Richardson. «The impact of invasive alien Prosopis species (mesquite) on native plants in different environments in South Africa,» South African Journal of Botany, vol. 97, p. 25-31, 2015.

[12] Zehnsdorf, Andreas, Andreas Hussner, Frank Eismann, Helmut Rönicke, Arnulf Melzer. «Management options of invasive Elodea nuttallii and Elodea canadensis,» Limnologica, vol. 51, pp. 110-117, 2015.

[13] Ho, Dang P., Huu Hao Ngo, Wenshan Guo. «A mini review on renewable sources for biofuel,» Bioresource Technology, vol. 169, p. 742-749, 2014.

[14] Gupta, Vijai K., Ravichandra Potumarthi, Anthonia O'Donovan, Christian P. Kubicek, Gauri Dutt Sharma, Maria G. Tuohy. «Bioenergy Research: An Overview on Technological Developments and Bioresources,» Bioenergy Research: Advances and Applications, pp. $23-47,2014$

[15] Swain, Manas Ranjan, Jyoti Mishra, Hrudayanath Thatoi. «Bioethanol Production from Sweet Potato (Ipomoea batatas L.) Flour using CoCulture of Trichoderma sp. and Saccharomyces cerevisiae in SolidState Fermentation,» Brazilian Archives of Biology and Technology, vol. 56, n 2, pp. 171-179, 2013

[16] Mishima, , D., M. Kuniki, K. Sei, S. Soda, M. Ike, M. Fujita. «Ethanol production from candidate energy crops: Water hyacinth (Eichhornia crassipes) and water lettuce (Pistia stratiotes L.),» Bioresource Technology, vol. 99, pp. 2495-2500, 2008.

[17] O'Sullivana, Cathryn, Beth Rounsefell, Alistair Grinham, William Clarke, James Udy. «Anaerobic digestion of harvested aquatic weeds: water hyacinth (Eichhornia crassipes), cabomba (Cabomba Caroliniana) and salvinia (Salvinia molesta),» Ecological Engineering 36 (2010) 1459-1468, vol. 36, nº 10, pp. 1459-1468, 2010.

[18] Nigam, Poonam Singh, Anoop Singh. «Production of liquid biofuels from renewable resources,» Progress in Energy and Combustion Science, vol. 37, pp. 52-68, 2011.

[19] Jaramillo F., María Consuelo, Luisa Fernanda Zapata O., Tatiana Marulanda L. «Fitorremediación de mercurio a partir de Elodea sp.,» Ing. USBMed, vol. $6, \mathrm{n}^{\circ} 2$, pp. 42-45, 2015

[20] Nigam, J.N. «Bioconversion of water-hyacinth (Eichhornia crassipes) hemicellulose acid hydrolysate to motor fuel ethanol by xylosefermenting yeast,» Journal of Biotechnology , vol. 97, pp. 107-116, 2002.

[21] Saha, Pradip, A. C. Baishnab, F. Alam, M. R. Khan, A. Islam «Production of bio-fuel (bio-ethanol) from biomass (pteris) by fermentation process with yeast,» Procedia Engineering, vol. 90, pp. 504-509, 2014.

[22] Cuervo, Laura, Jorge Luis Folch, Rosa Estela Quiroz. «Lignocelulosa Como Fuente de Azúcares Para la Producción de Etanol.» BioTecnología, vol. 13, nº 3, pp. 11-25, 2009.

[23] Singh, Anita, Punita Sharma, Alok Kumar Saran, Namita Singh, Narsi R. Bishnoi. «Comparative study on ethanol production from pretreated sugarcane bagasse using immobilized Saccharomyces cerevisiae on various matrices,» Renewable Energy, vol. 50, pp. 488-493, 2013.

[24] Best, Elly P. H. and J. H. A. Dassen. «A Seasonal Study of Growth Characteristics and the Levels of Carbohydrates and Proteins in Elodea 
Ingenierías USBMed, Vol. 8, No. 1, Enero-Junio 2017

nuttallii, Polygonum amphibium and Phragmites a Ustralis,» Aquatic Botany, vol. 28, pp. 353-372, 1987.

[25] Huanga, Wenmin, Hui Shaoa, Wei Li, Hongsheng Jianga, Yuanyuan Chen. «Article en Press. Effects of urea on growth and photosynthetic metabolism of two aquatic plants (Cabomba caroliniana A. Gray and
Elodea nuttallii (Planch.) H. St. John),» Aquatic Botany, vol. xxx, pp. xxx-xxx, 2016.

[26] Xiao, Lin, Liuyan Yang, Yi Zhang, Yufei Gu, Lijuan Jiang, Boqiang Qin. «Solid state fermentation of aquatic macrophytes for crude protein extraction,» Ecological Engineering , vol. 35, p. 1668-1676, 2009. 